\title{
Indicadores de salud mental en mujeres de Zacatecas con pareja migrante ${ }^{1}$
}

Mental health indicators of Zacatecas migrant partner women

\author{
Georgina Lozano Razo \\ María Dolores García Sánchez \\ Oliva Erendira Luis Delgado \\ Javier Zavala Rayas \\ Silvia del Carmen Miramontes Zapata \\ Juan Martín Sánchez Bautista²
}

\section{Resumen}

El objetivo de la siguiente investigación fue conocer si existen diferencias en los puntajes con respecto a los indicadores de depresión, hostilidad, sensibilidad interpersonal, somatización y ansiedad entre mujeres con pareja migrante que habitan en varias cabeceras municipales del estado de Zacatecas. Para ello se trabajó con una muestra total de 146 mujeres, habitantes de las cabeceras municipales de: Jerez, Francisco R. Murguía, Monte Escobedo, General Pánfilo Natera, Pinos y Villa Hidalgo. Todas ellas con pareja migrante en EE.UU. Se les aplicó la forma abreviada del cuestionario Symptom Assessment-45 Questionnaire (SA-45) (Sandín, Valiente, Chorot, Santed y Lostao, 1997), el cual es un instrumento de autoinforme de síntomas psicopatológicos. En la presente investigación se aplicaron las siguientes subescalas: depresión, hostilidad, sensibilidad interpersonal, somatización y ansiedad. Se aplicó una prueba no paramétrica y se obtuvieron diferencias estadísticamente significativas en la subescala de ansiedad, siendo las mujeres de la cabecera municipal General Pánfilo Natera quienes presentan un rango promedio más alto (84.89) y por lo tanto mayores niveles de ansiedad.

\section{Abstract}

The aim of this research was to determine the differences in the score for mental health indicators of depression, hostility, interpersonal sensitivity, somatization and anxiety among women with a migrant couple residing in various municipalities of the state of Zacatecas. The sample were 146 women, residents of six municipalities of the state of Zacatecas (Jerez, Francisco R. Murguia, Monte Escobedo, General Panfilo Natera, Pinos and Villa Hidalgo). All of their couples migrated to the U.S. They were given the Symptom Assessment-45 Questionnaire (SA-45) (Sandin, Valiente, Chorot, Sante and Lostao, 1997), which is a self-reported psychopathological symptoms instrument. In this study the following subscales were administered: depression, hostility, interpersonal sensitivity, somatization and anxiety. A nonparametric test was conducted from which statistically significant differences were obtained in the anxiety subscale: women from General Pánfilo Natera produced the highest average rank (84.89) and therefore the highest levels of anxiety.

\footnotetext{
${ }^{1}$ Investigación realizada con apoyo de Fondo Mixto CONACyT-Gobierno del estado de Zacatecas Convocatoria 2009-01, en colaboración con los CA: Migración y Desarrollo y Salud y Sociedad.

2 Universidad Autónoma de Zacatecas. Ágora José González Echeverría. C.P. 99000. Fresnillo, Zacatecas. Teléfono y fax (493) 93345 01. Correo electrónico: glozraz@yahoo.com.mx
}

Palabras clave: migración, mujeres, indicadores, salud mental, pareja migrante.

Keywords: migration, women, indicators, mental health, migrant couples. 


\section{Introducción}

El estudio de la migración como fenómeno social, es abordado por disciplinas científicas que pretenden dar respuesta a requerimientos económicos, políticos, jurídicos, demográficos, étnicos y culturales. Por ejemplo, se han realizado estudios sobre la salud mental de los que emigran (Finch, Hummer, Kol y Vega (2001), Grzywacs, Hovey, Seligman, Arcury y Quandt (2006), Goodkind, Gonzáles, Malcoe y Espinosa (2008) y Dunn y O'Brien (2009). Sin embargo, en México es poco común encontrar investigaciones referentes a los que permanecen en el lugar de origen, entre las que destacan están las de Salgado de Snyder (1996), AguileraGuzmán, Carreño y Juárez (2004), Polanco y Jiménez (2006), Oliva, León y Rivera (2007), Ramírez (2008).

Meza y Cuéllar (2009) realizaron en el estado de Michoacán entrevistas a profundidad con 55 mujeres, esposas de migrantes, hallando que si bien las mujeres describen a sus esposos como trabajadores, responsables, buena gente, alegres, hombres valientes que sufren y dan su vida por enviarles dinero a sus hijos y a ellas, también expresan emociones negativas de dolor, tristeza, soledad y falta de apoyo cuando los maridos no están en México.

Por su parte, Salgado de Snyder y Díaz (1994) aplicaron una escala a mujeres, que mide sintomatología depresiva, encontrando puntajes altos en dicha escala, por lo que concluye que las mujeres compañeras de migrantes se ven afectadas por la ausencia del cónyuge. Esto en parte puede deberse a que la migración de los hombres, hace que las mujeres asuman roles y actividades que antes estaban destinadas a los varones (Loza, Vizcarra, Lutz y Quintanar, 2007).

De acuerdo con McGuire y Martin (2007) las mujeres que permanecen en su lugar de origen a la espera de sus compañeros migrantes, sufren de tristeza, sobre todo ligada a la incertidumbre de no saber cuándo se podrá dar la reunificación familiar.
Los procesos de migración se han acelerado debido a las condiciones de pobreza, la falta de oportunidades así como la búsqueda de una mejor condición de vida. Para el año 2003, existían 191 millones de personas migrantes en el mundo, de los cuales un 60 por ciento residían en países desarrollados. El 65 por ciento era económicamente activo y un 48 por ciento mujeres. Aproximadamente un 3.5 por ciento de la población mundial vive en un país diferente al lugar donde nació; debido a esto la migración internacional se encuentra en una proporción que tiende a crecer y que comprende un nuevo desafío para las sociedades, los gobiernos y sobre todo para los servicios de salud (Organización Panamericana de la Salud [OPS] y Organización Mundial de la Salud [OMS], 2003).

Se estima que el número total de migrantes internacionales en todo el mundo, en 2010, asciende a 214 millones de personas. Entre 2005 y 2010 esa cifra ha permanecido relativamente estable como porcentaje de la población mundial, registrándose sólo un $0,1 \%$ de aumento (Departamento de Asuntos Económicos y Sociales de las Naciones Unidas [ONU DAES], 2009).

Con relación a la migración México-EE.UU., el Department of Economic and Social Affairs [DAES] (2002), reportó que antes del año 2000, la cantidad de inmigrantes de origen mexicano hacia los EE. UU. se multiplicó unas cien veces: para 1900 se calcula la presencia de 103 mil mexicanos, mientras que, para 2002, se estima en 9.5 millones; no obstante, el $90 \%$ de esta escalada ocurrió en las últimas tres décadas. El número de mexicanos radicados en el vecino país equivale a más del $8 \%$ de la población total de México y a $3 \%$ de la de aquel país.

La migración México-EE.UU. ha tenido un crecimiento exponencial en el curso de las últimas dos décadas, que se acentúa paradójicamente, con la puesta en marcha del TLCAN (Tratado de Libre Comercio América del Norte). Ello ha posicionado a México como el princi- 
pal emisor de migrantes al país vecino, que a su vez constituye el principal receptor de inmigrantes del mundo (Consejo Nacional de Población [Conapo], 2004).

De acuerdo con el Instituto Nacional de Estadística, Geografía e Informática [INEGI] (2010), en el año 2000, había 9, 023756 de personas nacidas en México que estaban residiendo en Estados Unidos, de los cuales 4, 977 486 eran hombres y 4, 046270 eran mujeres. Para el año 2008, había 11, 657266 de emigrantes mexicanos en EE.UU., siendo 6, 497 339 hombres y 5, 159927 mujeres.

Por otra parte, Zacatecas junto con Michoacán, Guanajuato y Jalisco, cuentan con una larga tradición migratoria, con un grado de intensidad muy alto. Respecto a la migración internacional de Zacatecas, el número de migrantes hacia Estados Unidos entre 1995 y 2000 fue de 66,790 , siendo el $76.3 \%$ hombres y el $23.7 \%$ mujeres, lo que constituye el $4.09 \%$ de migrantes respecto al total nacional, y representa un despoblamiento en 42 de sus 58 municipios (Delgado y Rodríguez, 2001). De acuerdo al II Conteo de Población y Vivienda, realizado por el INEGI, para el periodo 2000 a 2005, el $0.8 \%$ de la población de la entidad zacatecana de 5 años y más, reside en Estados Unidos, el $72.85 \%$ de esa población está constituida por hombres y el $27.15 \%$ por mujeres.

Según Mines (2000), dos terceras partes de los trabajadores agrícolas mexicanos en los Estados Unidos emigran primero que sus esposas; según datos de la Encuesta del Departamento del Trabajo de los Estados Unidos (citada por el mismo investigador) existe una fuerte tendencia entre los migrantes hacia la reunificación familiar en el vecino país. Al parecer la historia de las migraciones se ha caracterizado por periodos de hombres solos seguidos por periodos de reunificación familiar. Esta situación pone de manifiesto que los hombres emigran antes que sus esposas a los Estados Unidos. En el momento actual, dadas las condiciones políticas y sociales del vecino país, parece ser que el periodo de reunificación familiar no se dará pronto. Este hecho puede explicar en parte el aumento de los hogares con jefatura femenina en México (INEGI, 2009).

Por otra parte, la pobreza se encuentra ligada al género, de los 1300 millones de pobres absolutos, más del 70 por ciento son mujeres; las mujeres del mundo tienen menos acceso al mercado laboral que los hombres, trabajan más horas, sufren una tasa de desempleo mayor y reciben sueldos inferiores. En los países en desarrollo las mujeres se encargan del 80 por ciento de los cultivos alimentarios, pero no tienen la titularidad de propiedad sobre la tierra (Estébanez, 2002).

Se ha documentado que la motivación primordial de la mayoría de hombres y mujeres migrantes es mejorar sus condiciones de vida y las de su familia por medio de un mayor ingreso económico en dólares. Sin embargo, las mujeres que permanecen a cargo del cuidado de la familia no disminuyen su dependencia económica respecto a su esposo migrante; en este sentido, de acuerdo con la Encuesta Nacional sobre la Dinámica de las Familias [ENDIFAM] (Instituto de Investigaciones Sociales, 2005), la mitad de las mujeres con pareja migrante declara tener que consultar a sus maridos para comprar algo para la casa, esto muestra la importancia que tienen las opiniones de los ausentes en la toma de decisiones de sus compañeras.

El fenómeno migratorio ha sido reconocido por sus repercusiones importantes en el funcionamiento psicológico y social de los que en él participan (Salgado de Snyder, 1996). Durante mucho tiempo los estudios llevados a cabo en comunidades rurales expulsoras de migrantes se habían enfocado casi exclusivamente a investigar el impacto de la migración desde una perspectiva histórica, antropológica, demográfica, económica y sociológica (Fonseca y Moreno, 1984). Sin embargo, desde hace varios años los investigadores se han planteado abordar el tema de la salud mental tanto de los migrantes como de sus familias que permane- 
cen en su lugar de origen. A nivel familiar, se ha propuesto que la larga ausencia del cónyuge y los cambios que ocurren en el núcleo familiar son fuentes de conflicto, tanto para el migrante como para la familia que permanece en su lugar de origen (Trigueros y Rodríguez, 1988). Debido a que las necesidades de la familia cambian cuando alguno de sus miembros emigra, las tensiones de los núcleos familiares son tan grandes que rompen su equilibrio y surgen así los problemas que afectan su funcionamiento individual, familiar y social, los que, a su vez, repercuten en su salud mental.

En lo que respecta a este último punto, un estudio realizado en nuestro país por MedinaMora et al. (2003), sobre prevalencia de desórdenes psiquiátricos en México, encontró que entre las edades de 18 a 29 años los trastornos más frecuentes son la fobia específica y la social, los desórdenes bipolares, trastornos de conducta, trastorno oposicionista, trastornos por déficit de atención, abuso y dependencia de sustancias. En las edades de 30 a 44 años se presentan los trastornos de ansiedad generalizada, agorafobia sin pánico y trastorno de ansiedad de separación. De 45 a 54 años los trastornos más comunes fueron los trastornos por estrés post traumático, trastorno de pánico, el abuso y dependencia al alcohol. En los mayores de 55 años la prevalencia más elevada fueron el trastorno depresivo mayor y la distimia.

De acuerdo a los indicadores sociodemográficos identificados en otro estudio, MedinaMora, Borges, Benjet, Lara y Berglund (2007), encontraron diferencias de género, ya que las mujeres tienen mayor riesgo de presentar trastornos de ansiedad y del estado de ánimo y los hombres tienen mayor riesgo de padecer trastornos por uso de sustancias y control de impulsos. Por su parte, los jóvenes son los que tienen más riesgos de presentar la mayoría de los trastornos y, quienes tienen menos escolaridad tienen mayor riesgo de trastornos por uso de sustancias.
En el área de la psicología se han realizado estudios que indican que la migración es una experiencia traumática que se manifiesta en el momento de la partida-separación del lugar de origen, o en el de llegada al sitio nuevo, desconocido y que incluye una gama de factores que provocan estrés (Grinberg, 1996). De acuerdo con Salgado de Snyder (1996), el otro componente del proceso migratorio son los miembros de la familia inmediata de los migrantes, la esposa y sus hijos. Las mujeres mexicanas que no emigran a Estados Unidos. con su pareja, ya sea por obligación o por decisión propia, les corresponde una doble carga: por una parte sus propias responsabilidades, y por la otra, las responsabilidades del esposo que emigra; generalmente dichas responsabilidades se refieren a mantener la estabilidad, la salud, y la unidad de la familia durante la ausencia del cónyuge. Esto implica un desgaste importante que repercute en su funcionamiento psicosocial y su estado general de salud mental.

Para 1996, lo que se sabía del funcionamiento psicosocial de las mujeres que no migran con sus parejas, era limitado, pero suficiente para ofrecer una perspectiva sobre las características de vida durante la ausencia de los esposos. La migración del cónyuge significa, por un lado, un aumento en el número de responsabilidades y preocupación por el bienestar del esposo en un país desconocido, y por otro, también representa la posibilidad de tener una mejor calidad de vida para ellas y sus hijos, por lo que a pesar de resentir la partida del cónyuge, generalmente apoyan su decisión (Salgado de Snyder, 1996).

Algunos estudios han reconocido que el proceso migratorio altera las relaciones con la pareja y que sus efectos pueden ser tanto positivos como negativos. Por un lado, están los que afirman que la migración genera desventajas en las mujeres; por ejemplo, haciéndolas más vulnerables a la explotación económica y sexual. Por el otro, se encuentran los que señalan que la migración representa beneficios para ellas; 
por ejemplo, les ayuda a potenciar algunos aspectos de sus vidas tales como el ejercicio de una mayor autonomía o empoderamiento, tanto en las que emigran como en las que permanecen en sus comunidades de origen (Timur, 2000). Para Ariza (2000), la migración no produce cambios de manera automática, repercutiendo en un mejoramiento o decadencia de la vida de las mujeres, sino que depende tanto de contextos específicos, como de la experiencia de cada mujer.

En una investigación publicada recientemente por Castro (2009) se reporta que entrevistó a 15 madres de familia esposas o compañeras de migrantes internacionales, cuyas edades oscilaban entre 21 y 72 años. Todas ellas a cargo del hogar y de los hijos mientras el esposo o compañero es trabajador migrante, documentado o indocumentado en Estados Unidos. En las entrevistas, la autora encontró que aunque hay el convencimiento de que es mejor estar temporalmente separados, la mayoría de las informantes relata eventos de depresión o depresión posparto relacionados estrechamente con la situación migrante: largas ausencias con el consecuente distanciamiento físico, y en muchos casos, con el paso de los años, alejamiento sentimental; partos sin apoyo o presencia del cónyuge o compañero, estrechez económica, entre otros.

Por otra parte, en la investigación realizada en Zacatecas por García y Pérez (2008), en las localidades de Tetillas (Río Grande), La Victoria (Pinos) y Tacoaleche (Guadalupe), encontraron que la ausencia del padre impacta de manera negativa en la familia, pues a decir de las mismas esposas, llegan a sufrir enfermedades nerviosas y depresión crónica, que no siempre quieren o pueden reconocer, en donde el 88.14 por ciento de las mujeres entrevistadas dijeron haber pasado por tristeza o depresión en más de una ocasión en los últimos seis meses, al momento de la entrevista.
En otra investigación realizada en Zacatecas por García, Lozano, Luis, Robledo y Zavala (2009) en los municipios de Juan Aldama, Miguel Auza y Tlaltenango, se entrevistaron a madres de familia con pareja migrante, quienes expresaron preocupación por la integración familiar debido a la ausencia del esposo, por la conducta rebelde de los hijos, en especial de los adolescentes; preocupación por la salud y bienestar físico y psicológico de los hijos, al tiempo que manifiestan la necesidad de contar con servicios especializados de atención a la salud mental en su lugar de residencia.

Es por ello, que en la presente investigación el objetivo es comparar indicadores de salud mental (depresión, hostilidad, sensibilidad interpersonal, somatización y ansiedad) en mujeres con pareja migrante, que habitan las siguientes cabeceras municipales del estado de Zacatecas: Jerez, Francisco R. Murguía, Monte Escobedo, General Pánfilo Natera, Pinos y Villa Hidalgo.

Por lo que las hipótesis a probar son las siguientes:

Hipótesis Nula: No existen diferencias estadísticamente significativas respecto a los puntajes correspondientes a los indicadores de depresión, hostilidad, sensibilidad interpersonal, somatización y ansiedad entre mujeres con pareja migrante que habitan en las cabeceras municipales de Jerez, Francisco R. Murguía, Monte Escobedo, General Pánfilo Natera, Pinos y Villa Hidalgo.

Hipótesis alternativa: Sí existen diferencias estadísticamente significativas respecto a los puntajes correspondientes a los indicadores de depresión, hostilidad, sensibilidad interpersonal, somatización y ansiedad entre mujeres con pareja migrante que habitan en las cabeceras municipales de Jerez, Francisco R. Murguía, Monte Escobedo, General Pánfilo Natera, Pinos y Villa Hidalgo. 


\section{Método}

Participantes: Se obtuvo una muestra total de 146 mujeres, habitantes de seis cabeceras municipales del estado de Zacatecas. Para obtener dicha muestra se utilizó un muestreo por conveniencia, ya que se determinó trabajar en dichas cabeceras municipales. Se buscó a mujeres que tuvieran a sus parejas viviendo en Estados Unidos y que accedieran a participar en la investigación. Respecto a las características generales de la muestra, la media más alta de edad la presentan las participantes de la cabecera municipal de Monte Escobedo (43.9 años), mientras que la más baja es para las mujeres de Pinos (35.3 años). En cuanto a la escolaridad, solamente cuatro de las 146 participantes cuentan con una carrera profesional y 13 el bachillerato, lo que indica que en su mayoría estas mujeres apenas han logrado estudiar el nivel básico.

Población meta: Habitantes de las cabeceras municipales de Jerez, Francisco R. Murguía, Monte Escobedo, General Pánfilo Natera, Pinos y Villa Hidalgo.

Unidad de análisis: Mujeres que tienen a sus parejas en EE.UU.

Instrumentos: Se aplicó la versión abreviada del cuestionario Symptom Assessment45 Questionnaire (SA-45) (Sandín, Valiente, Chorot, Santed y Lostao, 1997), el cual es un autoinforme de síntomas psicopatológicos, que consta de 45 reactivos, divididos en nueve subescalas: Depresión $(\mathrm{a}=.85)$, hostilidad $(\mathrm{a}=$ .83), sensibilidad interpersonal $(a=.84)$, somatización $(\mathrm{a}=.80)$, ansiedad $(\mathrm{a}=.84)$, psicoticismo $(\mathrm{a}=.63)$, obsesión-compulsión $(\mathrm{a}=.72)$, ansiedad fóbica $(a=.71)$ e ideación paranoide $(a=.71)$. El índice de fiabilidad de la escala es $a=.85$.

Las opciones de respuesta son cinco: nada, en absoluto, un poco presente, moderadamente, bastante y mucho o extremadamente. En la presente investigación, después de pilotear el instrumento se decidió trabajar únicamente con las siguientes escalas: depresión (sentirse triste), hostilidad (discusiones frecuentes), sen- sibilidad interpersonal (sentirse inferior), somatización (dolores musculares) y ansiedad (miedo repentino). De igual forma se modificaron las opciones de respuesta, debido a que las participantes mostraron dificultades para comprenderlas, presentándose las siguientes opciones: nada, poco y mucho.

Procedimiento: Para poder realizar la aplicación de los instrumentos de recolección de datos, se hicieron visitas a las cabeceras municipales ya mencionadas, se identificaron informantes claves, los cuales referían qué mujeres tenían a su pareja en EE. UU.; una vez localizadas, ellas mismas proporcionaban datos para ubicar a otras mujeres con dichas características.

\section{Análisis de datos}

Los datos del cuestionario se analizaron de manera cuantitativa a través del SPSS versión 16.0 para Mac, aplicando estadística descriptiva para los datos sociodemográficos y la prueba no paramétrica de Kruskal-Wallis para la comparación entre las cabeceras municipales. El análisis de varianza de una clasificación por rangos de Kruskal-Wallis, es una prueba útil para decidir si k muestras independientes son de poblaciones diferentes. La prueba supone que la variable en estudio tiene como base una distribución continua. Requiere, por lo menos, una medida ordinal de la variable (s) dependiente (s). La prueba de Kruskal-Wallis determina si la desigualdad entre las sumas de rangos es tan grande que probablemente no proceden de muestras tomadas de la misma población. La estadística usada en dicha prueba es $\mathrm{H}$, la cual está distribuida como chi cuadrada con gl= k-1. Para el caso que nos ocupa, las variables dependientes son los puntajes obtenidos en los indicadores: depresión, hostilidad, sensibilidad interpersonal, somatización y ansiedad. La variable independiente es el municipio de procedencia. La región de rechazo consiste en todos los valores de $\mathrm{H}$ tan grandes que la probabilidad asociada con su ocurrencia conforme a Ho es igual a o menor que $p=.05$. 


\section{Resultados}

El motivo de migración por parte de las parejas, en la mayoría de los casos, es por falta de empleo, aunque existen casos que lo hacen como una especie de tradición, "porque así lo hizo su papá, sus hermanos, sus tíos, en fin, la familia".

Más de la mitad de la muestra cuenta con un trabajo remunerado (52.41\%), actividad que tienen que combinar con el cuidado de los hijos; en algunos casos son ellas las que llegan a enviar dinero a sus parejas, debido a la escasez de trabajo en el país vecino. El $10.34 \%$ de la muestra no recibe dinero de sus parejas, mientras que el $24.13 \%$ de ellas, sólo a veces les envían (ver Tabla 1).

\section{Tabla 1}

Datos sociodemográficos de las mujeres con pareja migrante por cabecera municipal

\begin{tabular}{|c|c|c|c|c|c|c|c|c|c|c|c|c|}
\hline Variable & \multicolumn{2}{|c|}{ Jerez $(n=28)$} & \multicolumn{2}{|c|}{$\begin{array}{l}\text { Fco. R. Murguía } \\
\qquad(\mathrm{n}=28)\end{array}$} & \multicolumn{2}{|c|}{$\begin{array}{l}\text { Monte Esco- } \\
\text { bedo }(n=23)\end{array}$} & \multicolumn{2}{|c|}{$\begin{array}{l}\text { Pánfilo Natera } \\
\qquad(n=23)\end{array}$} & \multicolumn{2}{|c|}{ Pinos $(n=24)$} & \multicolumn{2}{|c|}{$\begin{array}{l}\text { Villa Hidalgo } \\
\qquad(n=20)\end{array}$} \\
\hline$X_{\text {de Edad }}$ & \multicolumn{2}{|c|}{36.8} & \multicolumn{2}{|c|}{36.4} & \multicolumn{2}{|c|}{43.9} & \multicolumn{2}{|c|}{37.1} & \multicolumn{2}{|c|}{35.3} & \multicolumn{2}{|c|}{39.8} \\
\hline Grupo de edad & $f$ & $\%$ & $f$ & $\%$ & $f$ & $\%$ & $f$ & $\%$ & $\bar{f}$ & $\%$ & $f$ & $\%$ \\
\hline Menos de 20 & - & - & - & - & - & - & - & - & 1 & 4.2 & 1 & 5.0 \\
\hline 20-30 años & 9 & 32.2 & 8 & 28.6 & 6 & 26.1 & 3 & 13.0 & 8 & 33.3 & 2 & 10.0 \\
\hline 31-40 años & 8 & 28.6 & 12 & 42.8 & 5 & 21.8 & 12 & 52.2 & 10 & 41.7 & 7 & 35.0 \\
\hline 41-50 años & 10 & 35.6 & 6 & 21.4 & 3 & 13.1 & 7 & 30.5 & 3 & 12.5 & 8 & 40.0 \\
\hline $51-60$ años & 1 & 3.6 & 2 & 7.2 & 5 & 21.6 & 1 & 4.3 & 2 & 8.3 & 1 & 5.0 \\
\hline 61 en adelante & - & - & - & - & 4 & 17.4 & - & - & - & - & 1 & 5.0 \\
\hline Escolaridad & $f$ & $\%$ & $f$ & $\%$ & $f$ & $\%$ & $f$ & $\%$ & $f$ & $\%$ & $f$ & $\%$ \\
\hline 1. N. & - & - & 1 & 3.6 & - & - & - & - & 1 & 4.2 & - & - \\
\hline 2. P.I. & 5 & 17.9 & 6 & 21.4 & 6 & 26.1 & 10 & 43.5 & 1 & 4.2 & 3 & 15.0 \\
\hline 3. P.C. & 13 & 46.4 & 8 & 28.6 & 7 & 30.5 & 4 & 17.4 & 5 & 20.8 & 8 & 40.0 \\
\hline 4. S.C. & 9 & 32.1 & 8 & 28.6 & 5 & 21.7 & 7 & 30.5 & 11 & 45.8 & 6 & 30.0 \\
\hline 5. P.C. & 1 & 3.6 & 2 & 7.1 & 5 & 21.7 & 1 & 4.3 & 6 & 25.0 & 2 & 10.0 \\
\hline 6. P.P.C. & - & - & 3 & 10.7 & - & - & - & - & - & - & 1 & 5.0 \\
\hline Motivo migración & $f$ & $\%$ & $f$ & $\%$ & $f$ & $\%$ & $f$ & $\%$ & $f$ & $\%$ & $f$ & $\%$ \\
\hline Falta de empleo & 13 & 46.4 & 22 & 78.6 & 17 & 73.9 & 16 & 69.6 & 19 & 79.2 & 11 & 55.0 \\
\hline Costumbre & 4 & 14.3 & 1 & 3.6 & 4 & 17.4 & 6 & 26.1 & 4 & 16.7 & 8 & 40.0 \\
\hline Mejorar ingreso & 7 & 25.0 & 5 & 17.9 & 1 & 4.3 & - & - & - & - & - & - \\
\hline Ella trabaja & $f$ & $\%$ & $f$ & $\%$ & $f$ & $\%$ & $f$ & $\%$ & $f$ & $\%$ & $f$ & $\%$ \\
\hline Sí & 14 & 57.1 & 11 & 39.3 & 14 & 60.9 & 11 & 47.8 & 6 & 25.0 & 12 & 60.0 \\
\hline No & 12 & 42.9 & 17 & 60.7 & 9 & 39.1 & 12 & 52.2 & 18 & 75.0 & 8 & 40.0 \\
\hline Pareja envía dinero & $f$ & $\%$ & $f$ & $\%$ & $f$ & $\%$ & $f$ & $\%$ & $f$ & $\%$ & $f$ & $\%$ \\
\hline Sí & 20 & 71.4 & 20 & 71.4 & 11 & 47.8 & 13 & 56.5 & 18 & 75.0 & 12 & 60.0 \\
\hline No & 3 & 10.7 & 1 & 3.6 & 6 & 26.1 & 2 & 8.7 & 3 & 12.5 & - & \\
\hline$A$ veces & 5 & 17.9 & 7 & 25.0 & 5 & 21.7 & 8 & 34.8 & 3 & 12.5 & 7 & 35.0 \\
\hline
\end{tabular}

Nota. Claves para escolaridad: N. Ninguna. P.I. Primaria incompleta. P.C. Primaria completa. S.C. Secundaria o técnica completa. P.C. Preparatoria completa. P.P.C. Profesional completa y/o posgrado. 


\section{Conclusiones}

En lo relativo a las subescalas del instrumento se obtuvieron medidas descriptivas como la media y las desviación estándar en cada uno de los municipios, mismas que se describen en la Tabla 2. Para poder apreciar con más detalle estos datos, se aplicó una prueba no paramétrica de comparación de grupos; en este caso se recurrió a la prueba de Kruskal-Wallis, para comprobar diferencias en las subescalas: Depresión ( $x 2=6,262, \mathrm{gl}=5, \mathrm{p}=, 282$ ); Hostilidad ( $x 2=6,24, \mathrm{gl}=5, \mathrm{p}=, 283)$; Sensibilidad interpersonal $(x 2=2,460, \mathrm{gl}=5, \mathrm{p}=, 783)$; Somatización ( $x 2=6,587, \mathrm{gl}=5, \mathrm{p}=, 253)$; Ansiedad $(x 2=13,283, g l=5, p=, 021)$. Se observa una diferencia estadísticamente significativa en la subescala de ansiedad, siendo las mujeres de la cabecera municipal de General Pánfilo Natera quienes presentan el rango promedio más alto (84.89), mientras que las mujeres de la cabecera municipal de Francisco R. Murguía presentan el rango promedio más bajo (49.75).
Es importante mencionar que los resultados de este estudio son datos preliminares de una investigación más amplia que está en proceso, por lo que encontramos, existen algunos limitantes respecto al tratamiento de los datos, puesto que se prevé realizar análisis estadísticos más exhaustivos.

En este primer acercamiento se encontraron diferencias significativas en los puntajes al indicador de ansiedad entre mujeres con pareja migrante que habitan en la cabecera municipal de General Pánfilo Natera, respecto a los otros municipios. Estas diferencias pueden deberse a varios factores, uno de ellos está relacionado a que el 52.2 por ciento de las participantes no tienen una actividad remunerada, ya que el 56.6 por ciento de ellas dependen de las remesas enviadas por su pareja y un 43.5 por ciento "no" y "a veces" reciben apoyo financiero, lo que posiblemente puede producir en ellas un aumento de ansiedad.

\section{Tabla 2}

Medidas descriptivas para las subescalas del SA-45

\begin{tabular}{|c|c|c|c|c|c|c|}
\hline Subescala & Jerez $(n=28)$ & $\begin{array}{l}\text { Fco. R. Mur- } \\
\text { guía }(n=28)\end{array}$ & $\begin{array}{l}\text { Monte Escobe- } \\
\text { do }(n=23)\end{array}$ & $\begin{array}{l}\text { Pánfilo Natera } \\
\quad(n=23)\end{array}$ & Pinos $(n=24)$ & $\begin{array}{l}\text { Villa Hidalgo } \\
\qquad(n=20)\end{array}$ \\
\hline \multirow{2}{*}{ Depresión } & $\bar{X}=11,18$ & $\bar{X}=10,89$ & $\bar{X}=11,82$ & $\bar{X}=12,26$ & $\bar{X}=11,08$ & $\bar{X}=13,10$ \\
\hline & D.S $=3.98$ & D.S $=3.46$ & D.S $=3.62$ & D.S $=3.03$ & D.S $=3.06$ & D.S $=3.52$ \\
\hline \multirow{2}{*}{ Hostilidad } & $\bar{X}=7,71$ & $\bar{X}=6,85$ & $\bar{X}=7,00$ & $\bar{X}=7,39$ & $\bar{X}=6,20$ & $\bar{X}=7,75$ \\
\hline & D. $S=2.80$ & D.S $=2.10$ & D.S $=2.35$ & D. $. S=2.65$ & D.S $=1.41$ & D.S $=3.90$ \\
\hline \multirow{2}{*}{$\begin{array}{l}\text { Sensibilidad } \\
\text { Interpersonal }\end{array}$} & $\bar{X}=7,22$ & $\bar{X}=6,50$ & $\bar{X}=6,47$ & $\bar{X}=6,56$ & $\bar{X}=6,87$ & $\bar{X}=6,95$ \\
\hline & D.S $=2.60$ & D.S $=2.20$ & D.S $=2.39$ & D.S $=2.38$ & D.S $=2.07$ & D.S $=1.79$ \\
\hline \multirow{2}{*}{ Somatización } & $\bar{X}=11,50$ & $\bar{X}=10,32$ & $\bar{X}=12,17$ & $\bar{X}=11,00$ & $\bar{X}=10,00$ & $\bar{X}=10,45$ \\
\hline & D.S $=3.59$ & D.S $=3.44$ & D.S $=3.77$ & D.S $=3.43$ & D.S $=3.59$ & $\mathrm{D} . \mathrm{S}=2.64$ \\
\hline \multirow{2}{*}{ Ansiedad } & $\bar{X}=8,84$ & $\bar{X}=7,21$ & $\bar{X}=8,21$ & $\bar{X}=9,30$ & $\bar{X}=8,66$ & $\bar{X}=9,05$ \\
\hline & D.S $=3.15$ & D.S $=2.88$ & D.S $=2.90$ & D.S $=2.85$ & D.S $=2.18$ & D.S $=2.60$ \\
\hline
\end{tabular}


En este sentido los resultados reportados por ENDIFAM (Instituto de Investigaciones Sociales, 2005) señalan que el hecho de recibir apoyo económico del compañero migrante no significa necesariamente recibir apoyo emocional. De acuerdo con Salgado de Snyder (1996), en general, las mujeres casadas con migrantes, presentan sentimientos de desesperanza por las condiciones a las que cotidianamente tienen que enfrentarse, también padecen niveles significativamente más altos de malestar psicológico generalizado, sintomatología depresiva, ansiedad y somatización, que los que presentan las mujeres de las mismas comunidades con características similares, pero cuyos esposos no han emigrado.
En relación a las variables sociodemográficas como la edad (de 31 a 40 años) y la escolaridad (nivel básico), la muestra de estudio presenta las mismas características que lo hallado en otras investigaciones (ENDIFAM, 2005; Leyva y Caballero, 2009). Dichos indicadores muestran que las mujeres compañeras de migrantes están en plenitud reproductiva y económicamente productiva. Sin embargo, se ha demostrado que a mayor edad de esta población, menor es el apoyo emocional recibido por parte de la pareja (ENDIFAM, 2005), lo cual puede repercutir en su bienestar psicológico. 


\section{Referencias}

Aguilera-Gúzman, R., Carreño, M. y Juárez, F. (2004). Características psicométricas de la CES-D en una muestra de adolescentes rurales mexicanos de zonas con alta tradición migratoria. Salud mental, 27(6), 57-66.

Ariza, M. (2000). Género y migración femenina. En D. Barrera y C. Oehmichen (Eds.). Migración y relaciones de género en México (pp. 3362). México: GIMTRAP, UNAM/IIA

Castro, S. A. (2009). Migración, cambios y procesos microsociales: La familia en el espacio social transnacional. En. M.E. Ramos (coord.). Migración e identidad: emociones, familia, cultura. Monterrey, Nuevo León, México: Fondo Editorial de Nuevo León.

Consejo Nacional de Población. (2004). Encuesta sobre migración en la frontera norte. México: Secretaría de Gobernación, Instituto Nacional de Migración, Colegio de la Frontera Norte y Consejo Nacional de Población.

Delgado, R. y Rodríguez, H. (2001). Los Dilemas de la Migración y el Desarrollo en Zacatecas: El Caso de la Región de alta migración internacional. Recuperado el 10 enero de 2010, de http://www.migracionydesarrollo.org/

Departamento de Asuntos Económicos y Sociales de las Naciones Unidas. (2009). Trends in International Migrant Stock: The 2008 Revision. Recuperado el 13 marzo de 2011, de http://www.un.org/esa/ population/migration/UN_MigStock_2008

Department of Economic and Social Affairs. (2002). International Migration Report 2002. New York: United Nations.

Dunn, M. \& O'Brien, K. (2009). Psychological Health and Meaning in Life: Stress, Social Support and Religious Coping in Latina/Latino Immigrants. Hispanic Journal of Behavioral Science, 31, 204. doi: 10.1177/0739986309334799.

Estebanéz, P. (2002). Exclusión social y salud. Barcelona: Editorial Icaria

Finch, B., Hummer, R., Kol, B. \& Vega, W. (2001). The Role of Discrimination and Acculturative Stress in the Physical Health of MexicanOrigin Adults. Hispanic Journal of Behavioral Science, 23, 399. doi: 10.1177/0739986301234004

Fonseca, O. y Moreno, L. (1984). Jaripo, pueblo de migrantes. Michoacán, México: Centro de Estudios de la Revolución Mexicana.

García, R. y Pérez, O. (2008). Un pasivo: Mujeres y niños en comunidades de alta migración internacional en Michoacán, Jalisco y Zacatecas, México. New York, EE. UU.: United Nations Children's Fund (UNICEF), Policy, Advocacy and Knowledge Management (PAKM), Division of Policy and Practice.

García, S. M., Lozano, R. G., Luis, D. O., Robledo, M. V. y Zavala, R. J. (2009). Indicadores y necesidades psicosociales en zonas migrantes (tradicional y emergentes) del estado de Zacatecas. Informe de Investigación.

Goodkind, J., Gonzales, M., Malcoe, L. \& Espinosa, J. (2008). The Hispanic Women's Social Stressor Scale : Understanding the Multiple Social Stressors of U.S.- and Mexico-born Hispanic Women. Hispanic Journal of Behavioral Sciences, 30, 200. doi: 10.1177/0739986308316178.

Grinberg, R. (1996). Migración y exilio, estudio psicoanalítico. Madrid: Ed. Biblioteca Nueva

Grzywacz, J., Hovey, J., Seligman, L., Arcury, T. \& Quandt, S. (2006) Evaluating Short-Form Versions of the CES-D for Measuring Depressive Symptoms Among Immigrants From Mexico. Hispanic Journal of Behavioral Science, 28, 404. doi: 10.1177/0739986306290645

Instituto Nacional de Estadística, Geografía e Informática IXXX. (2009). II Conteo de Población y Vivienda. Recuperado el 8 de agosto de 2009, de http://www.inegi.org.mx
Instituto Nacional de Estadística, Geografía e Informática XXX. (2010). Mujeres y hombres en México 2010. México: Instituto Nacional de las Mujeres e Instituto Nacional de Estadística, Geografía e Informática.

Leyva, R. y Caballero, M. (2009). Las que se quedan: contextos de vulnerabilidad a ITS y VIH/SIDA en mujeres compañeras de migrantes. México: Instituto Nacional de Salud Pública.

Loza, M., Vizcarra, I., Lutz, B. y Quintanar, E. (2007). Jefaturas de hogar. El desafío femenino ante la migración transnacional masculina en el sur del Estado de México. Migraciones Internacionales, 19(2). Recuperado el 3 de noviembre de 2010, de http://www.scielo.org. $\mathrm{mx} /$ scielo.php?pid=S1665-89062007000200002\&script=sci_arttext

McGuire, S. \& Martin, K. (2007). Fractured migrant families: Paradoxes of hope and devastation. Family \& Community Health, 30, 178-188.

Medina-Mora, M. E., Borges, G., Lara, M. C, Benjet, C., Blanco J. J., Flez B. C., Villatoro V. J., Rojas G. E., Zambrano R. J., Casanova, R. L., y Aguilar G. S. (2003). Prevalencia de trastornos mentales y uso de servicios: Resultados de la encuesta nacional de epidemiología psiquiátrica en México. Salud Mental, 26(4), 1-16.

Medina-Mora, M. E., Borges, G., Benjet C., Lara C. \& Berglund, P. (2007). Psychiatric disorders in México: lifetime prevalence in a nationally representative sample. British Journal of Psychiatry, 521-528. doi:10.1192/bjp.bp.106.025841.

Meza, L. y Cuéllar, M. (2009). La vulnerabilidad de los grupos migrantes en México. México: Universidad Iberoamericana.

Mines, R. (2000). Las pautas migratorias mexicanas al agro norteamericano: Separación familiar y desarrollo atorado. En R. García-Zamora y M. Padilla (Eds.). Los Retos Demográficos de Zacatecas en el Siglo XXI (pp. 49-56). Zacatecas, México: Secretaría Académica de la Universidad Autónoma de Zacatecas.

Oliva, L., León, D. y Rivera, E. (2007). La Emigración del adulto como factor de riesgo en la autoestima de los adolescentes. Enseñanza e Investigación en Psicología, 12(2), 359-366.

Organización Panamericana de la Salud y Organización Mundial de la Salud. (2003). Nuevas perspectivas para la acción de la OPS/OMS: globalización y salud [132 sesión del comité ejecutivo].

Polanco, G. y Jiménez, N. (2006). Familias Mexicanas Migrantes: Mujeres que esperan. Psicología Iberoamericana, 14(2), 53-56.

Instituto de Investigaciones Sociales. (2005). Encuesta Nacional sobre la Dinámica de las Familias 2005. Recuperado el 19 de octubre de 2009, de http://www.bdsocial.org

Ramírez, B. A. (2008, octubre 12). Las esposas de migrantes sufren abandono y miseria. El Siglo de Durango, p12.

Salgado de Snyder, V. N. y Díaz, N. (1994). Factores psicosociales y conducta sexual riesgosa para el sida en mujeres rurales involucradas en la migración México-Estados Unidos. Salud Reproductiva, I, 9-12.

Salgado de Snyder, V. N. (1996). Problemas psicosociales de la migración internacional. Salud Mental, 19(1): 53-59.

Sandín, B., Valiente, R., Chorot, P., Santed, M. y Lostao, L. (2008). SA-45: Forma abreviada del SCL-90. Picothema, 20(2), 290-296.

Timur, S. (2000). Cambios de tendencias y problemas fundamentales de la migración internacional: una perspectiva general de los programas de la UNESCO. Revista Internacional de Ciencias Sociales, 65, 25-35.

Trigueros, P. y Rodríguez, J. (1988). Migración y vida familiar en Michoacán: un estudio de caso. En G. López-Castro (Ed.). Migración en el occidente de México. Zamora, Michoacán, México: El Colegio de Michoacán 